\title{
Sauropus androgynus leaves for health benefits: hype and the science
}

\begin{abstract}
Sauropus androgynus (SA) is a medicinal plant with high antioxidant potential. The leaves of this plant have been traditionally used to treat certain diseases, for weight loss, and as vegetable dishes. SA leaves contain an adequate amount of macronutrients and having most of the micronutrients. The micronutrients are phenolic compounds, carotenoids, antioxidant vitamins, and minerals. SA leaves also contain most of the essential minerals, including sodium, potassium, calcium, phosphorus, iron, magnesium, copper, zinc, manganese, and cobalt. Fresh leaves of SA typically consist of 70\%-90\% moisture, 3\%-8\% protein, $1 \%-4 \%$ fat, $1 \%-2 \%$ fiber, and about $2 \%$ ash. The other percentage of the leaves is carbohydrate. In this review, the types and amounts of phenolic compounds, carotenoids, antioxidant vitamins, and minerals are presented. Antinutrients and heavy metals detected in SA leaves are also revealed. These compounds could have adverse health effects, such as heavy metal toxicity and induce lung injury. Bronchiolitis obliterans and obstructive ventilatory impairment of the patients are known to be caused by ingestion of uncooked or fresh SA leaves. SA leaves are the staple food in some of the developing nations that provide essential nutrients to the poor people. It also helps to maintain good health of these people. However, fresh consumption and over-consumption of SA leaves are not advisable. It may cause toxicity or lung injury. The antioxidative components of SA leaves have scientifically shown a vast health benefit to the human being, from test tubes to in vivo studies. However, extra precaution should be taken for minimizing the adverse health effect of intake of fresh SA leaves.
\end{abstract}

Keyword: Antioxidant; Health benefit; Intoxicant; Leaves; Nutrient; Sauropus androgynus 